\title{
BMJ Open DECAF score as a mortality predictor for acute exacerbation of chronic obstructive pulmonary disease: a systematic review and meta-analysis
}

\author{
Qiangru Huang, ${ }^{1,2}$ Chengying He, ${ }^{1,2}$ Huaiyu Xiong, ${ }^{1,2}$ Tiankui Shuai, ${ }^{1,2}$ \\ Chuchu Zhang, ${ }^{1,2}$ Meng Zhang, ${ }^{1,2}$ Yalei Wang, ${ }^{1,2}$ Lei Zhu, ${ }^{1,2}$ Jiaju Lu, ${ }^{1,2}$ \\ Liu Jian (1) 1,2
}

To cite: Huang Q, He C, Xiong $\mathrm{H}$, et al. DECAF score as a mortality predictor for acute exacerbation of chronic obstructive pulmonary disease: a systematic review and meta-analysis. BMJ Open 2020;10:e037923. doi:10.1136/ bmjopen-2020-037923

- Prepublication history and additional material for this paper are available online. To view these files, please visit the journal online ().

Received 21 February 2020 Revised 05 June 2020 Accepted 10 July 2020
Check for updates

(c) Author(s) (or their employer(s)) 2020. Re-use permitted under CC BY-NC. No commercial re-use. See rights and permissions. Published by BMJ.

${ }^{1}$ ICU, Lanzhou University First Affiliated Hospital, Lanzhou, Gansu, China

${ }^{2}$ The First Clinical Medical College, Lanzhou University, Lanzhou, Gansu, China

Correspondence to

Professor Liu Jian;

medecinliu@sina.com

\section{ABSTRACT}

Objectives This study was conducted to assess the association between the Dyspnea, Eosinopenia, Consolidation, Acidemia and Atrial Fibrillation (DECAF) scores and the prognosis of patients with acute exacerbation of chronic obstructive pulmonary disease (AECOPD), to evaluate the specific predictive and prognostic value of DECAF scores and to explore the effectiveness of different cut-off values in risk stratification of patients with AECOPD.

Design Systematic review and meta-analysis.

Participants Adult patients diagnosed with AECOPD (over 18 years of age).

Primary and secondary outcome measures Electronic databases, including the Cochrane Library, PubMed, the Embase and the WOS, and the reference lists in related articles were searched for studies published up to September 2019. The identified studies reported the prognostic value of DECAF scores in patients with AECOPD.

Results Seventeen studies involving 8329 participants were included in the study. Quantitative analysis demonstrated that elevated DECAF scores were associated with high mortality risk (weighted mean difference $=1.87$; $95 \% \mathrm{Cl} 1.19$ to 2.56). In the accuracy analysis, DECAF scores showed good prognostic accuracy for both inhospital and 30-day mortality (area under the receiver operating characteristic curve: $0.83(0.79-0.86)$ and 0.79 $(0.76-0.83)$, respectively). When the prognostic value was compared with that of other scoring systems, DECAF scores showed better prognostic accuracy and stable clinical values than the modified DECAF; COPD and Asthma Physiology Score; BUN, Altered mental status, Pulse and age $>65$; Confusion, Urea, Respiratory Rate, Blood pressure and age > 65 ; or Acute Physiology and Chronic Health Evaluation II scores.

Conclusion The DECAF score is an effective and feasible predictor for short-term mortality. As a specific and easily scored predictor for patients with AECOPD, DECAF score is superior to other prognostic scores. The DECAF score can correctly identify most patients with AECOPD as low risk, and with the increase of cut-off value, the risk stratification of DECAF score in high-risk population increases significantly.
Strengths and limitations of this study

- This study evaluated the effectiveness of Dyspnea, Eosinopenia, Consolidation, Acidemia and Atrial Fibrillation (DECAF) score on prognosis short-term mortality of patients with acute exacerbation of chronic obstructive pulmonary disease (AECOPD), and explored the effectiveness of different cut-off values in risk stratification of patients with AECOPD.

- In order to further evaluate the effectiveness of DECAF score, this study compared the prognostic effects of DECAF scores with other prognostic scores, such as Acute Physiology and Chronic Health Evaluation II; BUN, Altered mental status, Pulse and age $>65$; and Confusion, Urea, Respiratory Rate, Blood pressure and age $>65$.

- This study assessed DECAF scores by quantitative analysis and accuracy analysis.

- The data and analyses were difficult to obtain due to a lack of original studies reporting the value of DECAF scores for predicting long-term mortality and other adverse outcomes in patients with AECOPD.

- Although we analysed the source of heterogeneity through subgroup analysis, heterogeneity in the results should still be considered carefully.

\section{INTRODUCTION}

Acute exacerbation of chronic obstructive pulmonary disease (AECOPD) is characterised by the deterioration of respiratory symptoms beyond normal daily variations. ${ }^{1}$ AECOPD accounts for one in eight hospital admissions $^{2}$ and is associated with worsening lung function, health-related quality of life and mortality risk. The in-hospital mortality of patients with AECOPD ranges from $4.4 \%$ to $25 \%$. The survivors have a readmission rate of $25 \%-55 \%$, and $25 \%-50 \%$ of these patients have a high risk of death within 1 year. ${ }^{23}$

Prognostic score can provide a strong indicator for risk stratification and assist clinical management, including hospital at home 


\begin{tabular}{|c|c|}
\hline Variables & Score \\
\hline Dyspnoea & 1 \\
\hline $\begin{array}{l}\text { eMRCD } 5 \text { a (too breathless to leave the house } \\
\text { unassisted but independent in washing and/or } \\
\text { dressing) }\end{array}$ & 1 \\
\hline $\begin{array}{l}\text { eMRCD } 5 b \text { (too breathless to leave the house } \\
\text { unassisted and requires help with washing and } \\
\text { dressing) }\end{array}$ & 2 \\
\hline Eosinopenia (eosinophils <0.05×10\%/L) & 1 \\
\hline Consolidation & 1 \\
\hline Moderate or severe acidaemia $(\mathrm{pH}<7.3)$ & 1 \\
\hline $\begin{array}{l}\text { Atrial fibrillation (including history of paroxysmal } \\
\text { atrial fibrillation) }\end{array}$ & 1 \\
\hline Maximum DECAF score & 6 \\
\hline
\end{tabular}

DECAF, Dyspnea, Eosinopenia, Consolidation, Acidemia and Atrial Fibrillation; eMRCD, extended Medical Research Council Dyspnea score.

or early supported discharge for low-risk groups, and early escalation or appropriate palliation for high-risk groups. ${ }^{4} 5$ The Dyspnea, Eosinopenia, Consolidation, Acidemia and Atrial Fibrillation (DECAF) score is a risk stratification tool designed to predict risk of death in patients with AECOPD, ${ }^{6}$ and can be easily applied at the bedside to guide treatment, such as hospital at home for low-risk patients. ${ }^{7}$ The DECAF score uses indices routinely available at admission. The score includes five predictors, the strongest of which is stable state dyspnoea, measured by the extended Medical Research Council Dyspnea score (table 1). ${ }^{8}$ The DECAF score showed promising performance in derivative studies, and was superior to other prognostic tools for patients with AECOPD. ${ }^{6}$ In 2014, the UK National COPD Audit recommends that DECAF scores be recorded for patients with AECOPD. ${ }^{9}$ Subsequently, an increasing number of original studies conducted derivation, internal and external validation and implementation of the DECAF score. The prognostic value of DECAF score still needs to be further verified by the methods of systematic review and meta-analysis, which is essential to prove the generalisation of prognosis scores.

This systematic review and meta-analysis evaluated the association between DECAF scores and the prognosis of patients with AECOPD, assessed the specific predictive and prognostic value of DECAF scores and explored the effectiveness of different cut-off values in risk stratification of patients with AECOPD. To further assess the clinical value of DECAF scores, we compared the test to other commonly used prognostic scores, including the modified DECAF (m-DECAF; the Dyspnoea, Eosinopenia, Consolidation, Acidemia and Frequency of admission in AECOPD in the last year),${ }^{10} \mathrm{COPD}$ and Asthma Physiology Score (CAPS), ${ }^{11}$ BUN, Altered mental status, Pulse and age $>65$ (BAP-65) ${ }^{12}$ Confusion, Urea, Respiratory Rate,
Blood pressure and age $>65$ (CURB-65) ${ }^{13}$ and Acute Physiology and Chronic Health Evaluation II (APACHE II) scoring systems. ${ }^{14}$ Although these scores are not designed or proposed for AECOPD, they are still commonly used in clinical practice for the prediction and prognostic evaluation of patients with AECOPD. This study aimed to evaluate and validate the effectiveness of the DECAF score and improve the clinical course and outcome of patients with AECOPD.

\section{MATERIALS AND METHODS}

All methods of this systematic review and meta-analysis followed the Preferred Reporting Items for Systematic Reviews and Meta-Analyses (PRISMA) guidelines. ${ }^{15}$

\section{Data sources and searches}

The review authors searched for medical literature before September 2019. The research was conducted in electronic databases including the Cochrane Library, PubMed, the Excerpt Medica Database (Embase), the Web of Science (WOS) and the reference lists from review articles, irrespective of publication dates, status or language. The search was conducted with the following keywords: DECAF Score or Dyspnea, Eosinopenia, Consolidation, Acidemia and Atrial Fibrillation Score and AECOPD or Acute Exacerbations of Chronic Obstructive Pulmonary Disease. Search strategies used in the Cochrane Library, PubMed, Embase and WOS can be found in the online supplementary file: search strategies.

This meta-analysis included studies that met the following criteria:

1. Adult patients diagnosed with AECOPD (over 18 years of age).

2. The studies included the results of DECAF score prognoses in patients with AECOPD. Study information could be extracted into a $2 \times 2$ contingency table. AECOPD was diagnosed based on the latest reference standard in the original study, such as the Global Initiative for Chronic Obstructive Lung Disease (GOLD) guideline, which was defined as an acute event characterised by worsening of the patient's respiratory symptoms beyond normal day-to-day variations, leading to medication changes.

3. No publication date, status or language restrictions were applied. Clinical original articles were included, whereas secondary studies, conference abstracts, editorials and animal experiments were excluded.

\section{Study selection}

Two review authors ( $\mathrm{QH}$ and $\mathrm{HX}$ ) independently assessed the studies to be included based on the titles, abstracts and keywords. If a study was found to be relevant to our topic, at least two reviewers further evaluated the full text to determine whether it met the inclusion criteria. In the case of inconsistencies between the reviewers, a third reviewer (JL) was consulted. The authors consulted the original authors to further ensure the eligibility of a study, 


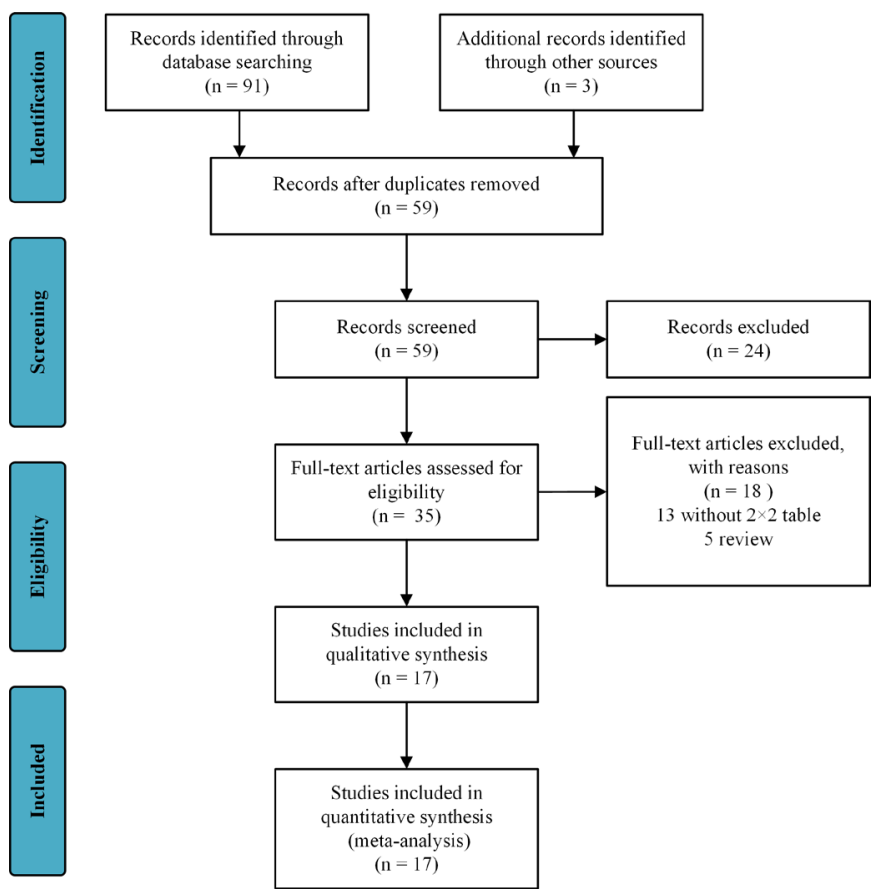

Figure 1 Preferred Reporting Items for Systematic Reviews and Meta-Analyses (PRISMA) flow diagram and exclusion criteria.

when additional information on the details of the results and methods or allocation concealment was needed. A study diagram was prepared to illustrate the entire literature research process and the selection of the studies (figure 1).

\section{Data extraction and quality assessment}

The data were independently extracted by two review authors (TS and CZ) and the resulting differences were resolved by a third reviewer $(\mathrm{CH})$. The extracted data included the lead author; publication year; the country of origin; the participant characteristics (age, sex and mortality rate); the statements for collection of DECAF; the optimal cut-off threshold in original study; values for sensitivity (SEN), specificity (SPE), true positive, true negative, false positive, false negative; and the area under the receiver operating characteristic (ROC) curve (AUC). If data were missing, a letter was written to the authors to request the data. If there was no response to the letter after 4 weeks, an email was sent. If there was no response to the email, estimates were made based on available data and used.

Two review authors (LJ and JL) independently applied the guidelines of the PRISMA statement ${ }^{16}$ to evaluate each involved study. The Quality Assessment of Diagnostic Accuracy Studies-2 was conducted by two independent authors (LJ and JL) to assess the quality and risk of bias for diagnostic or prognostic studies. ${ }^{17}$ In case of any inconsistency, all authors reach an agreement through discussion. The quality and risk of bias were assessed from two perspectives, including bias risk and applicability concerns, and evaluated from four aspects, including patient selection, index test, reference standard, and flow and timing.

\section{Data synthesis and analysis}

This study used Stata SE V.15.0 (StataCorp; College Station, TX, USA) to analyse the extracted data. Continuous variables are expressed as weighted mean differences (WMD) with a $95 \%$ CI.

The mixed bivariate random effects regression model was used to analyse and pool the diagnostic accuracy measurements across studies. ${ }^{18}$ To derive summary estimates, we plotted estimates of the observed SEN and SPE for each test in forest plots and hierarchical summary receiver operating characteristic (HSROC) curves derived from individual study results. ${ }^{19} 20$ These results were plotted using HSROC curves with 95\% confidence and prediction regions. Additionally, pooled SEN, SPE, diagnostic OR (DOR), positive likelihood ratio (PLR) and negative likelihood ratio (NLR) were calculated. ${ }^{21}$ The AUC was also calculated to show the prognostic performance of DECAF. In clinical practice, tests with AUC above 0.8 are considered to be very reliable. ${ }^{22}$

The heterogeneity of eligible studies was assessed by the Cochrane $Q$ test (significant heterogeneity was indicated by $\mathrm{p}<0.05$ ) and the $\mathrm{I}^{2}$ test (significant heterogeneity was indicated by $\left.\mathrm{I}^{2}>50 \%\right) .{ }^{23}$ If substantive heterogeneity $\left(\mathrm{I}^{2}\right.$ $>50 \%$ ) existed, subgroup analysis and SEN analysis were performed to analyse the sources of the heterogeneity. The $\alpha$ value was set to 0.05 .

To assess the heterogeneity from the threshold effect, the Spearman correlation coefficient between the logit of SEN and the logit of (1-specificity) was computed to assess the threshold effect on the prognostic accuracy of DECAF score. If the Spearman correlation coefficient was greater than or equal to $0.6(\mathrm{p}<0.05)$, there was a threshold effect. ${ }^{24}$ The Deeks funnel plot asymmetry test was used to assess for publication bias, when the included studies were greater than 10 studies. $^{25}$

\section{Patient and public involvement}

Patients and the public were not involved in the development of the research question, the outcome measures, the design or conduct of this systematic review. Patients and the public were not asked to advise on interpretation of results or to contribute to the writing or editing of this document.

\section{RESULTS}

\section{Study selection}

A flow chart of the study selection process (figure 1) was prepared according to the PRISMA guidelines. After reviewing the title and abstract, 35 articles were screened for full-text review. Among them, 18 articles failed to meet the inclusion criteria. Seventeen studies involving a total of 8329 participants met all of the criteria. ${ }^{69-40}$ Among them, Echevarria $e t a l^{2628}$ and Shi et $a l^{2729}$ each produced two articles from two different studies. 


\section{Study characteristics}

As for the AECOPD definition, all studies were defined by the GOLD criteria, which is defined as an acute event characterised by worsening of the patient's respiratory symptoms beyond normal day-to-day variations and leading to medication changes. ${ }^{41}$ All identified studies reported the results of DECAF scores for AECOPD prognosis. Among these studies, 15 reported the prognostic values of DECAF scores for in-hospital mortality ${ }^{6} 926282931-40$ and five studies reported 30-day mortality. ${ }^{27} 28303133$ The cut-off threshold for each study was retrospectively determined based on the ROC curve. For in-hospital mortality, the results of five studies were based on a cut-off value of $4,{ }^{928353739}$ four studies were based on a cut-off value of $3,{ }^{6} 323640$ three studies were based on a cut-off value of $2^{303338}$ and the other three studies did not report a cut-off threshold. ${ }^{1722} 25$ With regard to the collection of DECAF score, eight studies collected the score on admission, ${ }^{9} 273032-343840$ one reported that the collection was prespecified in the original study protocol, ${ }^{26}$ one was collected within 24 hours after admission, ${ }^{35}$ one recorded DECAF score as part of routine practice ${ }^{28}$ and the other six reported that the DECAF score was compiled based on admission data. ${ }^{6931} 363739$ As for other prognostic scores, five studies reported the prognostic value of CURB-65 scores, ${ }^{28} 30313335$ eight reported BAP-65 scores, ${ }^{28} 3031$ 33-37 five reported APACHE II scores, ${ }^{627-29} 40$ four reported CAPS scores ${ }^{62829} 40$ and three reported the prognostic value of m-DECAF scores ${ }^{9} 29$ for patients with AECOPD. A summary of the characteristics of the included studies is shown in table 2.

\section{Methodological quality and risk of bias}

Only one study was a case-control design without blinding statements, which could not prevent the occurrence of observer bias, thus the risk of bias was related high. ${ }^{35}$ All studies included patients diagnosed with AECOPD, and eight studies reported consecutive enrolment. ${ }^{6926-28303440}$ Most of studies included did not prespecify the cut-off value for risk stratification. Since the main outcome is the mortality of patients with AECOPD, for which the reference standard is survival or non-survival, all included studies met the low-risk criteria of the reference standard items. However, the included studies yielded different baseline characteristics in the included population, which affected patient selection, flow and timing. The quality and bias of each included study was shown in table 3, and the summary figures of risk of bias were shown in online supplementary figures S1 and S2.

\section{The quantitative analysis of DECAF scores in AECOPD}

Three studies referred to DECAF scores between the survivor group and the non-survivor group. The randomised effect model showed a significant increase in DECAF scores in the non-survivor group compared with the survivor group (WMD=1.87; 95\% CI 1.19 to 2.56; $\mathrm{p}<0.001$ ) (table 4). The results indicate that the elevated DECAF scores were associated with high mortality risk.
As shown in table 4 , four other scoring systems have been proven to indicate poor outcomes of AECOPD. Compared with the survivor group, the results showed that CURB-65 scores, BAP-65 scores, m-DECAF scores and APACHE II scores were increased in the non-survivor group (WMD $=0.69,95 \% \mathrm{CI}-0.08$ to $1.45, \mathrm{p}=0.078$; $\mathrm{WMD}=0.75,95 \% \mathrm{CI}-0.07$ to $1.56, \mathrm{p}=0.071$; $\mathrm{WMD}=1.74$, $95 \%$ CI 1.36 to $2.13, \mathrm{p}=0.001$; WMD $=5.24,95 \%$ CI 4.00 to $6.47, \mathrm{p}<0.001$, respectively). The results showed that increases in DECAF scores, m-DECAF scores and APACHE II scores were associated with a high risk of mortality in AECOPD, suggesting that DECAF scores have the potential to be a prognostic indicator for patients with AECOPD.

\section{Prognostic value of DECAF scores for AECOPD}

Seventeen studies reported the prognostic value of DECAF scores. The pooled SEN of DECAF scores for predicting mortality was $0.76\left(95 \%\right.$ CI 0.70 to $0.81 ; \mathrm{I}^{2}=45.24 \%$, $Q=29.22(\mathrm{p}=0.02))$ with an SPE of $0.76(95 \%$ CI 0.68 to $0.83 ; \mathrm{I}^{2}=96.99 \%, Q=531.44(\mathrm{p}<0.001)$; figure 2$)$. The PLR and NLR were 3.2 (95\% CI 2.4 to 4.1$)$ and 0.32 (95\% CI 0.27 to 0.37 ), respectively, and the DOR was 10 (95\% CI 8 to 13 ). The AUC of the HSROC was 0.82 (95\% CI 0.78 to 0.85 ; figure 3 ), indicating that the DECAF score had a reliable accuracy in predicting mortality for patients with AECOPD. Additionally, there was no significant difference in threshold effect (Spearman correlation coefficient $=0.467 ; \mathrm{p}=0.059$ ). No publication bias was found in Deeks funnel plot asymmetry test $(\mathrm{p}=0.74$; online supplementary figure S3).Fig. S3

\section{Subgroup analysis}

In predicting in-hospital mortality, the pooled SEN of the DECAF scores was 0.77 (95\% CI 0.70 to $0.82 ; \mathrm{I}^{22}=47.24 \%$, $\mathrm{p}=0.02$ ), the SPE was 0.76 (95\% CI 0.67 to $0.84 ; \mathrm{I}^{2}=96.5 \%$, $\mathrm{p}<0.001)$ and the AUC of the HSROC was $0.83(95 \%$ CI 0.79 to 0.86 ). For 30 -day mortality, the pooled SEN of the DECAF scores was 0.71 (95\% CI 0.53 to 0.84 ; $\left.\mathrm{I}^{2}=84.95 \%, \mathrm{p}<0.001\right)$, the SPE was $0.75(95 \%$ CI 0.58 to $\left.0.86 ; \mathrm{I}^{2}=98.37 \%, \mathrm{p}<0.001\right)$ and the AUC of the HSROC was 0.79 (95\% CI 0.76 to 0.83 ) (table 5 ).

The subgroup analyses were based on different cutoff values (table 5 ). For a cut-off value of 4 , five studies included 2550 participants who reported the prognostic value of DECAF. The pooled SEN of the DECAF scores was 0.75 (95\% CI 0.69 to $0.81 ; \mathrm{I}^{2}=0.00 \%, \mathrm{p}=0.61$ ), the SPE was 0.80 (95\% CI 0.68 to $\left.0.89 ; \mathrm{I}^{2}=95.84 \%, \mathrm{p}<0.001\right)$, the AUC of the HSROC was 0.76 (95\% CI 0.72 to 0.80 ), the PLR was 3.80 (95\% CI 2.20 to 6.60) and the NLR was 0.31 (95\% CI 0.23 to 0.41 ). Four studies included 1361 participants who reported the results of a cut-off value of 3 . The pooled SEN was 0.77 (95\% CI 0.70 to $0.82 ; \mathrm{I}^{2}=0.00 \%$, $\mathrm{p}=0.52$ ), the SPE was 0.76 (95\% CI 0.67 to $0.84 ; \mathrm{I}^{2}=29.09 \%$, $\mathrm{p}=0.24$ ), the AUC of the HSROC was 0.83 (95\% CI 0.79 to 0.86 ), the PLR was 3.20 (95\% CI 2.40 to 4.40 ) and the NLR was 0.31 (95\% CI 0.25 to 0.37 ). For a cut-off value of 2, three studies included 1002 participants who reported 


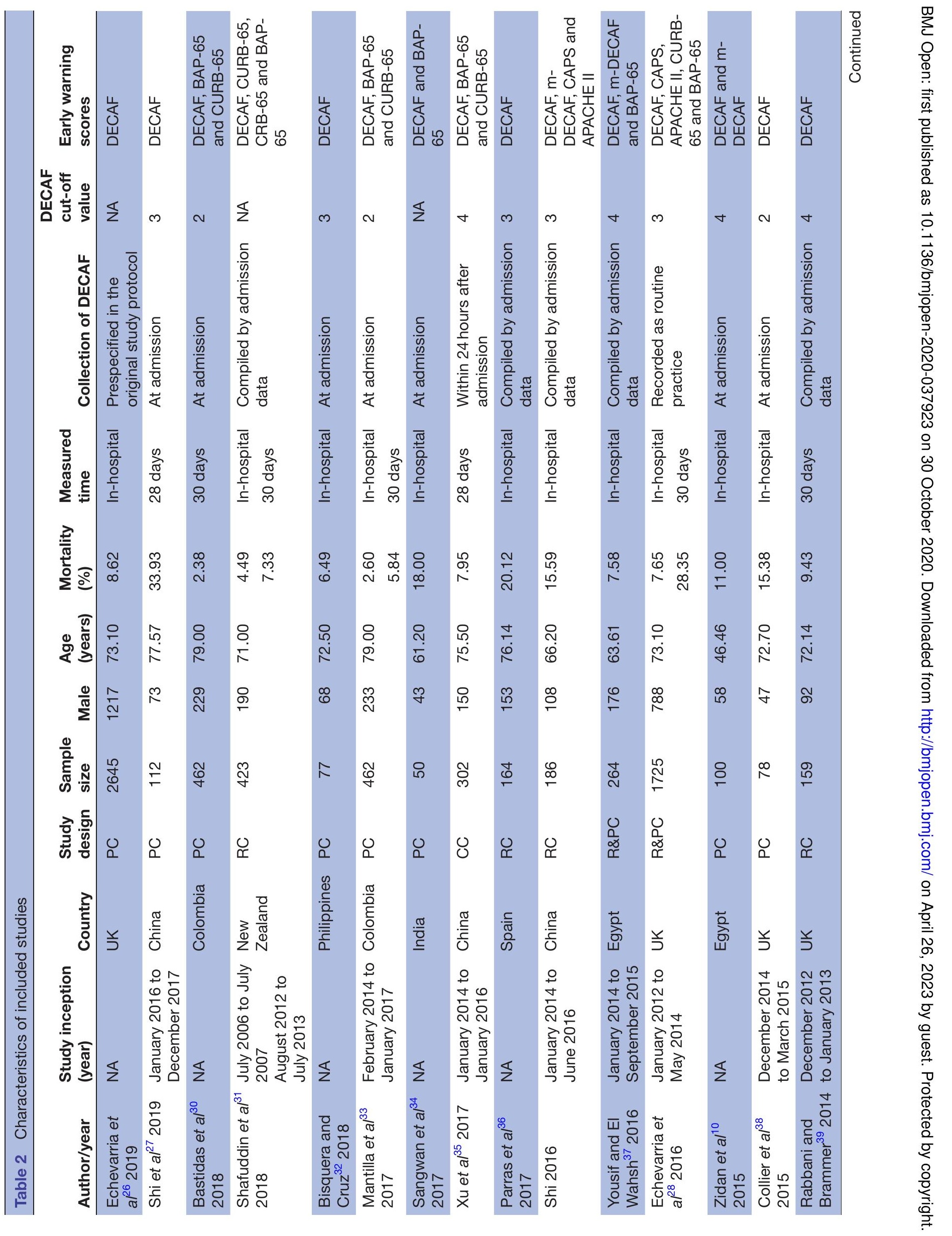




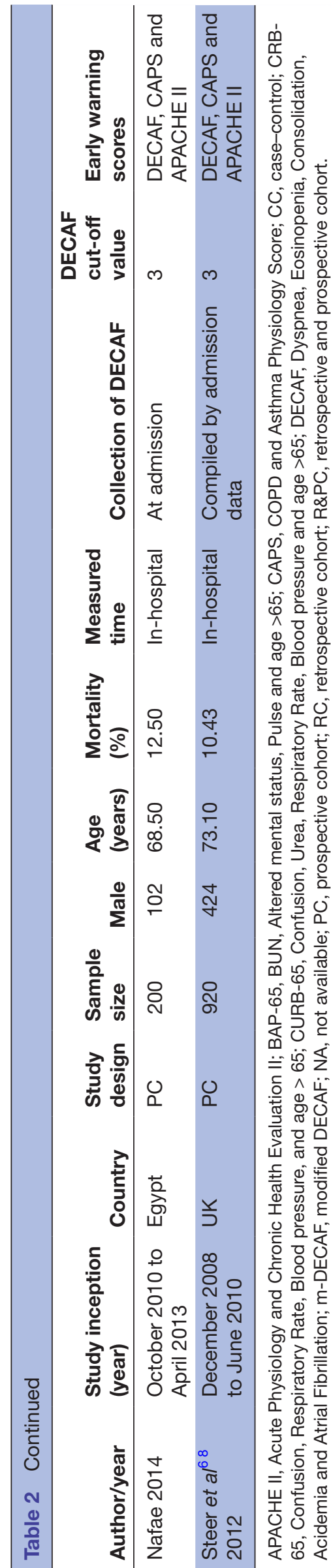

the results. The pooled SEN was 0.84 (95\% CI 0.68 to $\left.0.93 ; \mathrm{I}^{2}=0.00 \%, \mathrm{p}=0.52\right)$, the SPE was $0.53(95 \% \mathrm{CI} 0.50$ to $\left.0.56 ; \mathrm{I}^{2}=0.00 \%, \mathrm{p}=0.61\right)$, the AUC of the HSROC was 0.77 (95\% CI 0.73 to 0.80 ), the PLR was 1.80 (95\% CI 1.50 to $2.10)$ and the NLR was 0.31 (95\% CI 0.15 to 0.64$)$. The results of PLR and NLR at different cut-off values suggest that DECAF score can correctly identify most of patients with AECOPD as low risk, and with the increase of cut-off value, the risk stratification of DECAF score for high-risk population increased significantly.

\section{Other prognostic scores for patients with AECOPD}

In predicting the in-hospital mortality of patients with AECOPD, the pooled results showed that the SEN, SPE and AUC of the CURB-65 scores were 0.46, 0.92 and 0.73, respectively. The SEN, SPE and AUC of the BAP-65 scores were $0.70,0.50$ and 0.64 , respectively. The SEN, SPE and AUC of the APACHE II scores were 0.70, 0.65 and 0.72, respectively. The SEN, SPE and AUC of CAPS scores were $0.77,0.62$ and 0.75 , respectively, and the SEN, SPE and AUC of the m-DECAF scores were 0.84, 0.62 and 0.84, respectively (table 6 ).

When predicting the 30-day mortality in patients with chronic obstructive pulmonary disease (COPD), the pooled results showed that the SEN, SPE and AUC of the CURB-65 scores were $0.52,0.85$ and 0.53 , respectively. The SEN, SPE and AUC of the BAP-65 scores were 0.61, 0.57 and 0.62 , respectively. The SEN, SPE and AUC of the APACHE II scores were $0.68,0.73$ and 0.77 , respectively (table 7).

\section{DISCUSSION}

In stable COPD, prognostic indicators have been thoroughly investigated and tools to predict mortality risk, such as the body mass index, air-flow obstruction, dyspnea, and exercise capacity (BODE) score, ${ }^{41}$ have been well established. However, prognostic studies in patients with exacerbation requiring hospitalisation are limited and the predictors of mortality between stable disease periods and AECOPD periods seem to have little in common. ${ }^{42}$ In addition, the risk of mortality in patients with AECOPD is much higher than in patients with stable COPD. Thus, there is an urgent need for effective reliable clinical tools that can be used to inform clinicians and patients of the risk of death during exacerbation.

The current study conducted a systematic review and meta-analysis to characterise and evaluate DECAF scores predicting mortality in patients with AECOPD. Six potential scoring systems were evaluated by comparing survivor and non-survivor scores and prognostic accuracy. Quantitative analysis demonstrated that elevated DECAF scores were significantly associated with high mortality risk. In other potential scoring systems, compared with the survivor group, the results showed that only the m-DECAF and APACHE II scores increased in the nonsurvivor group. In the accuracy analysis, DECAF scores showed a reliable prognostic accuracy for both in-hospital 
Table 3 The Quality Assessment of Diagnostic Accuracy Studies-2 (QUADAS-2) for included studies

\begin{tabular}{|c|c|c|c|c|c|c|c|c|c|c|c|c|c|c|c|}
\hline \multirow[b]{2}{*}{ Studies } & \multicolumn{4}{|c|}{ Patient selection } & \multicolumn{3}{|c|}{ Index test } & \multicolumn{3}{|c|}{$\begin{array}{l}\text { Reference } \\
\text { standard }\end{array}$} & \multicolumn{4}{|c|}{ Flow and timing } & \multirow[b]{2}{*}{ Scores } \\
\hline & 1 & 2 & 3 & 4 & 5 & 6 & 7 & 8 & 9 & 10 & 11 & 12 & 13 & 14 & \\
\hline Echevarria et $\mathrm{a}^{26}$ & Y & Y & $u$ & Low & $\mathrm{Y}$ & Y & Low & Y & $u$ & Low & $\mathrm{Y}$ & Y & Y & Low & 12 \\
\hline Shi et $a l^{27}$ & $\mathrm{Y}$ & $\mathrm{Y}$ & Y & Low & $\mathrm{Y}$ & $U$ & Unclear & $Y$ & $U$ & Low & $U$ & $Y$ & Y & Low & 10 \\
\hline Bastidas et $a^{30}$ & $U$ & Y & $U$ & Unclear & $\mathrm{Y}$ & $U$ & Unclear & Y & $U$ & Low & $\mathrm{Y}$ & Y & Y & Low & 8 \\
\hline Shafuddin et $\left.a\right|^{31}$ & $U$ & $\mathrm{Y}$ & Y & Unclear & $\mathrm{Y}$ & $U$ & Unclear & $\mathrm{Y}$ & $U$ & Low & $\mathrm{Y}$ & $Y$ & $\mathrm{~N}$ & High & 7 \\
\hline Bisquera and $\mathrm{Cruz}^{32}$ & $u$ & Y & $u$ & Unclear & $Y$ & U & Low & $Y$ & U & Low & $\mathrm{Y}$ & $Y$ & U & Unclear & 7 \\
\hline Mantilla et $a /^{33}$ & $U$ & $\mathrm{Y}$ & U & Unclear & $\mathrm{Y}$ & U & Low & $\mathrm{Y}$ & $U$ & Low & $\mathrm{Y}$ & $Y$ & $\mathrm{Y}$ & Low & 9 \\
\hline Sangwan et $\left.a\right|^{34}$ & Y & Y & Y & Unclear & $\mathrm{Y}$ & U & Low & Y & U & Low & $\mathrm{Y}$ & $Y$ & U & Unclear & 9 \\
\hline Xu et $\left.a\right|^{35}$ & $U$ & $\mathrm{~N}$ & Y & High & $\mathrm{N}$ & $U$ & High & $\mathrm{Y}$ & $\mathrm{N}$ & High & $U$ & $Y$ & $\mathrm{Y}$ & Unclear & 4 \\
\hline Parras et $a^{36}$ & U & Y & Y & Unclear & Y & U & Low & $Y$ & U & Low & $U$ & $Y$ & Y & Low & 9 \\
\hline Shi 2016 & $U$ & Y & $\mathrm{Y}$ & Unclear & $\mathrm{Y}$ & $U$ & Low & $Y$ & $Y$ & Low & $U$ & $Y$ & $\mathrm{Y}$ & Low & 10 \\
\hline $\begin{array}{l}\text { Yousif and El } \\
\text { Wahsh }^{37}\end{array}$ & $U$ & $\mathrm{Y}$ & $\mathrm{Y}$ & Unclear & $\mathrm{Y}$ & U & Unclear & Y & $U$ & Low & $U$ & $Y$ & Y & Low & 8 \\
\hline Echevarria et $a^{28}$ & Y & Y & Y & Low & $\mathrm{Y}$ & $U$ & Low & $\mathrm{Y}$ & Y & Low & $\mathrm{Y}$ & $\mathrm{Y}$ & $\mathrm{Y}$ & Low & 13 \\
\hline Zidan et $a 1^{10}$ & Y & Y & Y & Unclear & $Y$ & U & Low & Y & U & Low & $Y$ & $Y$ & Y & Low & 12 \\
\hline Collier et $a^{38}$ & U & Y & U & Unclear & $\mathrm{Y}$ & $U$ & Low & $Y$ & U & Low & $U$ & $Y$ & $U$ & Unclear & 6 \\
\hline $\begin{array}{l}\text { Rabbani and } \\
\text { Brammer }^{39}\end{array}$ & U & $\mathrm{Y}$ & $U$ & Unclear & $U$ & $U$ & Unclear & $Y$ & $U$ & Low & $U$ & $Y$ & $\mathrm{Y}$ & Low & 6 \\
\hline Nafae 2014 & Y & $\mathrm{Y}$ & $Y$ & Low & $Y$ & $U$ & Low & $Y$ & $U$ & Low & $Y$ & $Y$ & $Y$ & Low & 12 \\
\hline Steer et $a l^{68}$ & Y & $Y$ & $Y$ & Low & $Y$ & U & Unclear & $Y$ & U & Low & $Y$ & $Y$ & $Y$ & Low & 11 \\
\hline
\end{tabular}

QUADAS-2 criteria: (1) Was a consecutive or random sample of patients enrolled? (2) Was a case-control design avoided?

(3) Did the study avoid inappropriate exclusions? (4) Could the selection of patients have introduced bias? (5) Were the index test results interpreted without knowledge of the results of the reference standard? (6) If a threshold was used, was it prespecified? (7) Could the conduct or interpretation of the index test have introduced bias? (8) Is the reference standard likely to correctly classify the target condition? (9) Were the reference standard results interpreted without knowledge of the results of the index tests? (10) Could the reference standard, its conduct, or its interpretation have introduced bias? (11) Was there an appropriate interval between index test and reference standard? (12) Did all patients receive the same reference standard? (13) Were all patients included in the analysis? (14) Could the patient flow have introduced bias?

$\mathrm{N}, \mathrm{No}$, represents negative answer for the corresponding question; $\mathrm{U}$, Unclear, that is, the information provided in the individual studies was insufficient to answer the corresponding question; Y, Yes, represents certain answer for the corresponding question.

and 30-day mortality. When the prognostic value was compared with other prognostic scores, DECAF scores showed better prognostic accuracy and stable clinical value in predicting the in-hospital mortality and 30-day mortality of patients with AECOPD. The results showed that for the different cut-off values of DECAF score, as the cut-off value increased, the SEN decreased and the SPE escalated. The results of PLR and NLR at different cutoff values suggest that DECAF score can correctly identify most patients with AECOPD as low risk, and with the

Table 4 The quantitative analysis of scores in AECOPD mortality

\begin{tabular}{lllllrr}
\hline Variables & Studies $(\mathbf{n})$ & Patients $(\mathbf{n})$ & WMD & 95\% $\mathbf{C l}$ & P value \\
\hline DECAF & 3 & 600 & 1.87 & 1.19 to 2.56 & $<0.001$ \\
CURB-65 & 2 & 414 & 0.69 & -0.08 to 1.45 & 0.078 \\
BAP-65 & 2 & 414 & 0.75 & -0.07 to 1.56 & 0.071 \\
Modified DECAF & 2 & 298 & 1.74 & 1.36 to 2.13 & 0.001 \\
APACHE II & 2 & 298 & 5.24 & 4.00 to 6.47 & $<0.001$ \\
\hline
\end{tabular}

AECOPD, acute exacerbation of chronic obstructive pulmonary disease; APACHE II, Acute Physiology and Chronic Health Evaluation II; BAP-65, BUN, Altered mental status, Pulse and age >65; CURB-65, Confusion, Urea, Respiratory Rate, Blood pressure and age >65; DECAF, Dyspnea, Eosinopenia, Consolidation, Acidemia and Atrial Fibrillation; WMD, weighted mean difference.; 


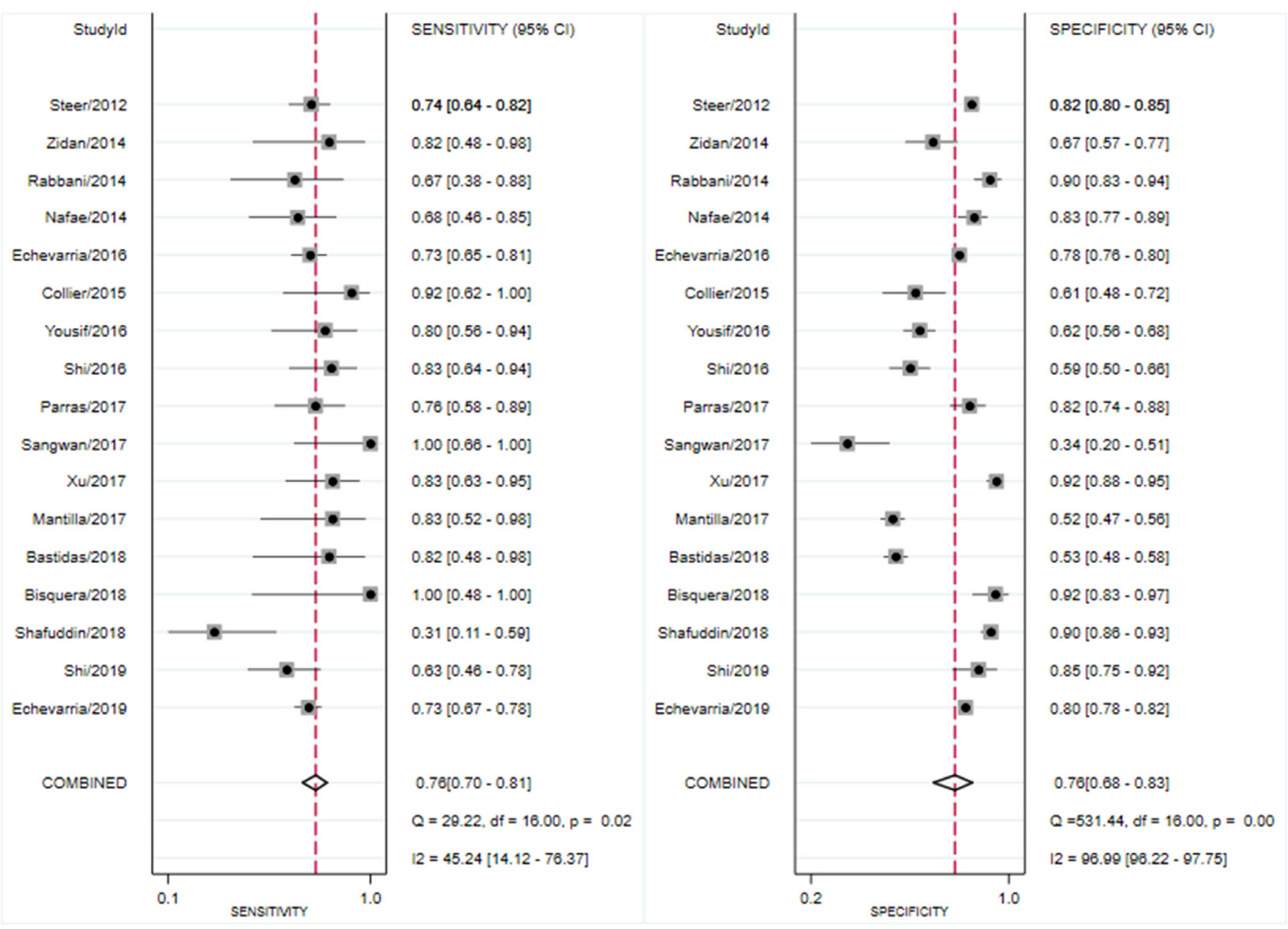

Figure 2 Forest plot of sensitivity and specificity of Dyspnea, Eosinopenia, Consolidation, Acidemia and Atrial Fibrillation (DECAF) for the prediction of mortality in acute exacerbation of chronic obstructive pulmonary disease (AECOPD).

increase of cut-off value, the risk stratification of DECAF score for high-risk population increased significantly.

The DECAF scores increased significantly in the nonsurvivor group. This suggests that elevated DECAF scores have the potential to stratify a high-risk population from low-risk patients. The m-DECAF and APACHE II scores had a similar relationship, which indicates that scoring systems have potential to aid clinical decisions in risk stratification. However, the CURB-65 and BAP-65 scores did not show statistical differences between the survivor and non-survivor groups. Although studies have shown that CURB-65 and BAP-65 can be effective tools for predicting mortality, ${ }^{43}$ based on the results of this current study, we speculate that the potential prognostic value of CURB-65 and BAP-65 is relatively low.

The DECAF score is an effective predictor of mortality and can be easily scored at the bedside using indices routinely available at admission. ${ }^{6}$ In clinical practice, test with AUC greater than 0.8 is considered to be very reliable. $^{22}$ The results showed that the AUC of the DECAF scores was 0.83 for predicting in-hospital mortality and 0.79 for short-term mortality ( 30 days). This indicates that the DECAF test can be used as a promising prognosis tool with satisfactory SEN and SPE for patients with AECOPD.

In a randomised controlled trial and economic evaluation study of DECAF implementation, the low-risk patients (DECAF 0 or 1) selected by DECAF were more cost-effective than the usual care, mainly manifested in a fivefold reduction in the median of 90 days of hospitalisation. ${ }^{7}$ The study showed that the DECAF score was easily applied at the bedside to guide treatment, and about twice as many patients were eligible compared with earlier models. ${ }^{7}$ It was safe, clinically effective, costeffective to use DECAF score at home in low-risk patients, and preferred by most patients. ${ }^{7}$

Mortality rates vary between clinical settings and cohorts. In this study, the mortality rate of patients in the included studies ranged from $2.38 \%$ to $33.93 \%$. This largely reflects differences in baseline characteristics, especially in the proportion of patients admitted from institutional care and with coexisting pneumonia. ${ }^{12} 28$ In addition, this also partly leads to choosing different cut-off values. To illustrate the relationship between the cut-off values for risk stratification, subgroup analyses 


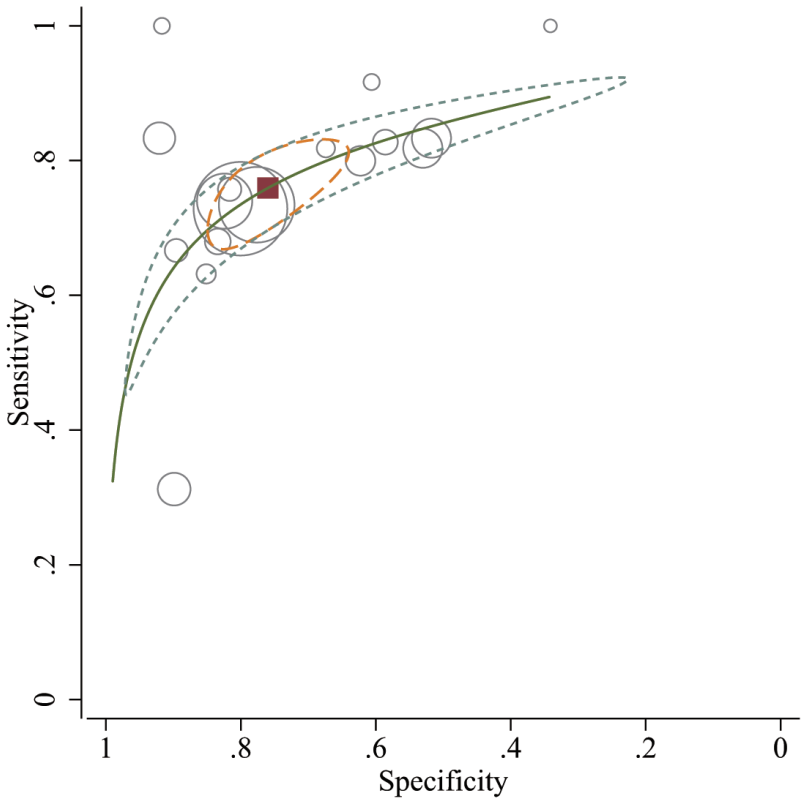

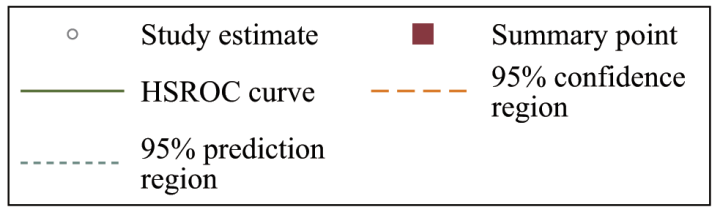

Figure 3 Hierarchical summary receiver operating characteristic curve for evaluating the prognostic value of mortality of Dyspnea, Eosinopenia, Consolidation, Acidemia and Atrial Fibrillation (DECAF) in acute exacerbation of chronic obstructive pulmonary disease (AECOPD). The HSROC, hierarchical summary receiver operating characteristic (HSROC) curve was conducted which plots sensitivity versus specificity. All studies were presented as a circle and plotted with the HSROC curve. The summary point (red box) indicates that the summary sensitivity was 0.76 and the summary specificity was 0.76 . The summary results are displayed as the $95 \%$ confidence region and $95 \%$ prediction region in the HSROC curve plot. The size of the marker is scaled according to the total number of patients in each study.

were performed. For cut-off values from 2 to 4 , the SEN decreased from 0.84 to 0.75 and the SPE increased from 0.53 to 0.80 . With an increase in the cut-off value, SPE increased significantly. Under the premise of ensuring SEN, improving SPE can effectively reduce the number of false positives and improve the clinical application value of a prognostic score.

In clinical practice, the greater the PLR value, the greater the likelihood of true positive when the test result is positive; the smaller the NLR value, the greater the likelihood of true negative when the test result is negative. PLR is more important in stratification of high-risk groups, while NLR is more important in low-risk groups. From the results, the NLR was very small, 0.31 , which indicated that the DECAF score could correctly identify most patients with AECOPD as a low-risk group. For the cut-off value from 2 to 4 , the PLR value increased from 1.80 to

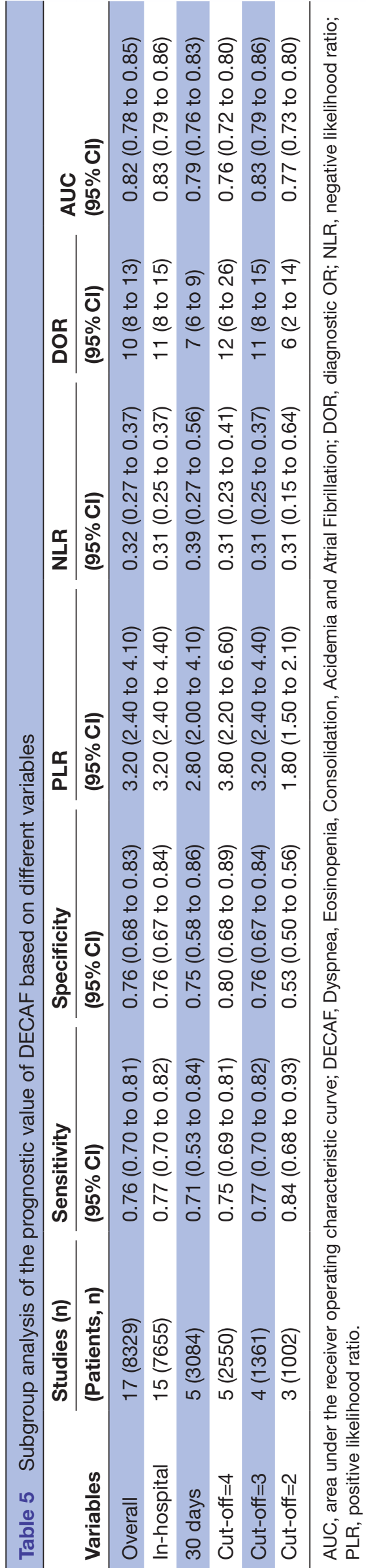



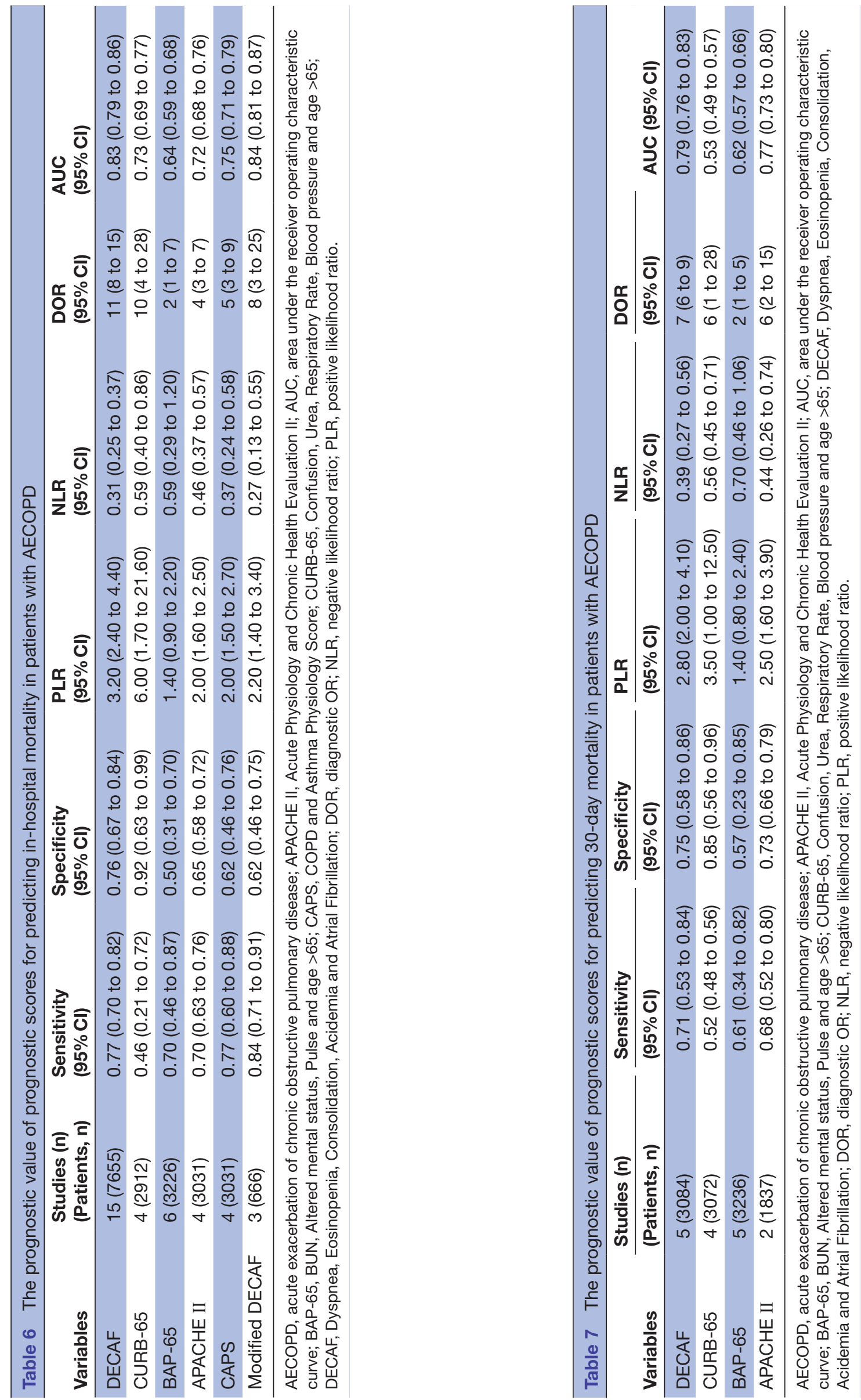
3.80 , indicating that with the increase of the cut-off value, the risk stratification of the DECAF score in high-risk groups increased significantly.

The CURB-65 and BAP-65 tests can also be easily scored on admission. ${ }^{44}$ However, according to the results of this study, the CURB-65 and BAP-65 scores had low prognostic values for predicting in-hospital and 30-day mortality, which were consistent with the lack of statistical difference in CURB-65 and BAP-65 scores between survivors and non-survivors.

APACHE II uses point scores based on the initial values of 12 routine physiological measurements, age and previous health status to provide a general measure of disease severity. ${ }^{45}$ APACHE II is not a specific predictor for AECOPD but is still commonly used in clinical practice to predict mortality in patients with AECOPD. ${ }^{46}$ Based on our results, APACHE II scores showed no superiority to DECAF scores in prognostic accuracy, SEN or SPE. In addition, it contains cumbersome test items, thus increasing the workload of clinicians in clinical practice. For patients with AECOPD, the APACHE II test may not be the preferred early warning scoring system.

As for the m-DECAF, Zidan et at attempted to replace the atrial fibrillation item in the DECAF test with admission frequency for AECOPD during the last year and named the revision the m-DECAF. They concluded that the m-DECAF test was more sensitive and specific in predicting in-hospital mortality during acute exacerbation of COPD than the DECAF test. However, there was no significant difference between the two scores, ${ }^{9}$ which was consistent with the results of this current study. In addition, only three studies reported the predictive value of m-DECAF test for in-hospital mortality in patients with AECOPD, and no study reported the effectiveness of the test in terms of 30-day mortality. Therefore, more evidence is needed to evaluate the prognostic value of m-DECAF scores and further compare the clinical value between DECAF scores and m-DECAF scores.

Examination of prognostic scores can contribute to clinical management, early risk stratification and the prevention of poor outcomes, as well as monitoring during treatment. ${ }^{47}$ Clinicians are constantly seeking predictors of mortality for patients with AECOPD. As a promising predictor, DECAF scores can be used in a variety of hospital settings to accurately stratify mortality risk. As a specific and easily scored predictor for patients with AECOPD, DECAF is superior to other prognostic scores in predicting short-term mortality. From the results of different cut-off values, the DECAF score showed a promising potential. It can correctly identify most patients with AECOPD as low-risk group, which is related to the reduction of in-hospital stay. Compared with the meta-analyses of interventions, including randomised controlled trials, those including diagnostic studies have more publication bias. ${ }^{48}$ Excluding studies that do not have sufficient data may lead to publication and reporting bias. Therefore, the prognostic value of DECAF may be overestimated. As for the significant degree of heterogeneity, we conducted a subgroup analysis to explore the source of the heterogeneity. The subgroup analysis revealed that the heterogeneity was mainly derived from the choice of cut-off value. When the cut-off value was 2, 3 or 4, the heterogeneity of SEN decreased to 0 . However, the heterogeneity of SPE was still substantive when the cut-off value was 4 . This largely reflects differences in the baseline characteristics of the patient selection. The biases between included studies can also lead to heterogeneity. The DECAF score needs to be collected at admission or prespecified in the original study protocol. However, the collection of DECAF score varied between the included studies, which may result in variable performance of DECAF. In addition, different included studies yielded different baseline characteristics in the included population, which affected patient selection and also led to the different selection of cut-off value between studies.

This meta-analysis had some limitations. First, the data and analyses were difficult to obtain due to a lack of original studies reporting the value of DECAF scores for predicting long-term mortality and other adverse outcomes in patients with AECOPD. Further studies are needed for validation. Second, it was difficult to obtain raw data for each of the included studies, which limited us to determining the optimal DECAF cut-off point for predicting AECOPD. Third, because of the lack of original research comparing DECAF with other predictive scores, we can only compare the predictive value of DECAF and other predictive scores to patients with AECOPD in general. With the increase of related original research, it is possible to further explore the effectiveness of different prognostic scores in risk stratification of patients with AECOPD. In addition, although the source of heterogeneity was analysed by subgroup analysis, heterogeneity in the results should still be considered carefully.

\section{CONCLUSION}

In conclusion, the results of this systematic review and meta-analysis indicated that the DECAF score was an effective and feasible predictor of short-term mortality in patients with AECOPD. As a specific and easily scored predictor for patients with AECOPD, DECAF scores are superior to other prognostic scores. The DECAF score can correctly identify most patients with AECOPD as low risk, and with the increase of cut-off value, the risk stratification of DECAF score for high-risk population increased significantly.

Acknowledgements The authors gratefully acknowledge the support of The First Clinical Hospital of Lanzhou University, The First Clinical Medical College of Lanzhou University, Evidence-based Medicine Center of Lanzhou University and all the authors who participated in this study.

Contributors JL, QH, CH, MZ, CZ, HX and TS participated in the design of the project, conducted the literature review and participated in the analysis. $\mathrm{QH}$ and YW wrote this paper. LZ and JL were responsible for the statistical analysis and participated in data interpretation. JL was the principal investigator for the project. All authors approved the final version of the article.

Funding The authors remain independently of any funding influence. 
Competing interests None declared.

Patient consent for publication Not required.

Provenance and peer review Not commissioned; externally peer reviewed.

Data availability statement All data relevant to the study are included in the article or uploaded as supplementary information. All data generated or analysed during this study are included in this published article and its supplementary information files, and no unpublished data are available.

Open access This is an open access article distributed in accordance with the Creative Commons Attribution Non Commercial (CC BY-NC 4.0) license, which permits others to distribute, remix, adapt, build upon this work non-commercially, and license their derivative works on different terms, provided the original work is properly cited, appropriate credit is given, any changes made indicated, and the use is non-commercial. See: http://creativecommons.org/licenses/by-nc/4.0/.

\section{ORCID iD}

Liu Jian http://orcid.org/0000-0002-1825-571X

\section{REFERENCES}

1 Rodriguez-Roisin R. Toward a consensus definition for COPD exacerbations. Chest 2000;117:398S-401.

2 Johannesdottir SA, Christiansen CF, Johansen MB, et al. Hospitalization with acute exacerbation of chronic obstructive pulmonary disease and associated health resource utilization: a population-based Danish cohort study. J Med Econ 2013;16:897-906.

3 de Miguel-Díez J, Jiménez-García R, Hernández-Barrera V, et al. Trends in hospital admissions for acute exacerbation of COPD in Spain from 2006 to 2010. Respir Med 2013;107:717-23.

4 Wildman MJ, Sanderson C, Groves J, et al. Predicting mortality for patients with exacerbations of COPD and asthma in the COPD and asthma outcome study (CAOS). QJM 2009;102:389-99.

5 Doll H, Miravitlles M. Health-Related QOL in acute exacerbations of chronic bronchitis and chronic obstructive pulmonary disease: a review of the literature. Pharmacoeconomics 2005;23:345-63.

6 Steer J, Gibson J, Bourke SC. The DECAF score: predicting hospital mortality in exacerbations of chronic obstructive pulmonary disease. Thorax 2012;67:970-6.

7 Echevarria C, Gray J, Hartley T, et al. Home treatment of COPD exacerbation selected by DECAF score: a non-inferiority, randomised controlled trial and economic evaluation. Thorax 2018;73:713-22.

8 Steer J, Norman EM, Afolabi OA, et al. Dyspnoea severity and pneumonia as predictors of in-hospital mortality and early readmission in acute exacerbations of COPD. Thorax 2012;67:117-21.

9 Stone RA, Holzhauer-Barrie J, Lowe D, et al. Copd: who cares matters. National chronic obstructive pulmonary disease (COPD) audit programme: clinical audit of COPD exacerbations admitted to acute units in England and Wales 2014, 2015.

10 Zidan MH, Rabie AK, Megahed MM, et al. The usefulness of the DECAF score in predicting hospital mortality in acute exacerbations of chronic obstructive pulmonary disease. Egypt $J$ Chest Dis Tuberc 2015;64:75-80.

11 Wildman MJ, Harrison DA, Welch CA, et al. A new measure of acute physiological derangement for patients with exacerbations of obstructive airways disease: the COPD and asthma physiology score. Respir Med 2007;101:1994-2002.

12 Shorr AF, Sun X, Johannes RS, et al. Validation of a novel risk score for severity of illness in acute exacerbations of COPD. Chest 2011:140:1177-83.

13 Lim WS, van der Eerden MM, Laing R, et al. Defining community acquired pneumonia severity on presentation to hospital: an international derivation and validation study. Thorax 2003;58:377-82.

14 Jacobs S, Chang RW, Lee B. One year's experience with the APACHE II severity of disease classification system in a general intensive care unit. Anaesthesia 1987;42:738-44.

$15 \mathrm{Ge}$ L, Tian J-H, Li Y-N, et al. Association between prospective registration and overall reporting and methodological quality of systematic reviews: a meta-epidemiological study. J Clin Epidemiol 2018;93:45-55.

16 Liberati A, Altman DG, Tetzlaff J, et al. The PRISMA statement for reporting systematic reviews and meta-analyses of studies that evaluate health care interventions: explanation and elaboration. $J$ Clin Epidemiol 2009;62:e1-34.

17 Whiting PF, Rutjes AWS, Westwood ME, et al. QUADAS-2: a revised tool for the quality assessment of diagnostic accuracy studies. Ann Intern Med 2011;155:529-36.
$18 \mathrm{Kim} \mathrm{KW}$, Lee J, Choi SH, et al. Systematic review and meta-analysis of studies evaluating diagnostic test accuracy: a practical review for clinical Researchers-Part I. General guidance and tips. Korean J Radiol 2015;16:1175-87.

19 Lee J, Kim KW, Choi SH, et al. Systematic review and meta-analysis of studies evaluating diagnostic test accuracy: a practical review for clinical Researchers-Part II. statistical methods of meta-analysis. Korean J Radiol 2015;16:1188-96.

20 Rutter CM, Gatsonis CA. A hierarchical regression approach to meta-analysis of diagnostic test accuracy evaluations. Stat Med 2001;20:2865-84.

$21 \mathrm{Chu} \mathrm{H}$, Cole SR. Bivariate meta-analysis of sensitivity and specificity with sparse data: a generalized linear mixed model approach. J Clin Epidemiol 2006;59:1331-2.

22 Memon MA, Faryal S, Brohi N, et al. Role of the DECAF score in predicting in-hospital mortality in acute exacerbation of chronic obstructive pulmonary disease. Cureus 2019;11:e4826.

23 Higgins J, Green S. Cochrane Handbook for systematic reviews of interventions version 5.1.0. Cochrane Collaboration, 2008.

24 Devillé WL, Buntinx F, Bouter LM, et al. Conducting systematic reviews of diagnostic studies: didactic guidelines. BMC Med Res Methodol 2002;2:9.

25 Deeks JJ, Macaskill P, Irwig L. The performance of tests of publication bias and other sample size effects in systematic reviews of diagnostic test accuracy was assessed. J Clin Epidemiol 2005;58:882-93.

26 Echevarria C, Steer J, Bourke SC. Comparison of early warning scores in patients with COPD exacerbation: DECAF and news score. Thorax 2019;74:941-6.

27 Shi Q-F, Sheng Y, Zhu N, et al. The v-DECAF score can predict 90-day all-cause mortality in patients with COPD exacerbation requiring invasive mechanical ventilation. Clin Respir $J$ 2019;13:438-45.

28 Echevarria C, Steer J, Heslop-Marshall K, et al. Validation of the DECAF score to predict hospital mortality in acute exacerbations of COPD. Thorax 2016;71:133-40.

29 Shi QF, Sheng Y, Wang SY. Comparison of four score modes in prognosis assessment of AECOPD patients with respiratory failure. Journal of Practical Medicine 2017;33:242-5.

30 Bastidas AR, Hincapie Diaz G, Mantilla Cardozo B, et al. Validity curb 65, BAP 65, DECAF for predicting outcomes in exacerbation of COPD. American Journal of Respiratory and Critical Care Medicine 2018;197

31 Shafuddin E, Chang CL, Hancox RJ. Comparing severity scores in exacerbations of chronic obstructive pulmonary disease. Clin Respir J 2018;12:2668-75.

32 Bisquera RR, Cruz BOD. Prognostic utility of the DECAF score to predict in-hospital mortality among patients with acute exacerbation of chronic obstructive pulmonary disease admitted at Chinese General Hospital. Respirology 2018;23:128-9.

33 Mantilla BM, Ramírez CA, Valbuena S, et al. Saturación de oxígeno/ fracción inspirada de oxígeno como predictor de mortalidad en pacientes Con exacerbación de EPOC atendidos en El Hospital Militar central. Acta Medica Colombiana 2017;42:215-23.

34 Sangwan V, Chaudhry D, Malik R. Dyspnea, eosinopenia, consolidation, acidemia and atrial fibrillation score and BAP-65 score, tools for prediction of mortality in acute exacerbations of chronic obstructive pulmonary disease: a comparative pilot study. Indian J Crit Care Med 2017;21:671-7.

35 MM X, SY Y, Zhang TT. Evaluation of the three scores to assess the severity of chronic obstructive pulmonary disease exacerbation. Journal of Tianjin Medical University 2017;23:530-3.

36 Parras AMV, Bautista CL, Chica GP, et al. Evaluation of DECAF, CURB-65 and BAP-65 scales as predictor of mortality risk in acute exacerbation of COPD in a retrospective cohort. European Respiratory Journal 2017;50.

37 Yousif M, El Wahsh RA. Predicting in-hospital mortality in acute exacerbation of COPD: is there a golden score? Egyptian Journal of Chest Diseases and Tuberculosis 2016;65:579-84.

38 Collier L, David T, Craig C, et al. P45 Practical use of the DECAF score: Can we improve outcomes in acute exacerbation of COPD admissions? Thorax 2015;70:A98.1-A98.

39 Rabbani B, Brammer P. P151 can the Decaf score be used to guide prognosis after an acute admission for COPD exacerbation? Thorax 2014:69:A139-40.

40 Nafae R, Embarak S, Gad DM. Value of the DECAF score in predicting hospital mortality in patients with acute exacerbation of chronic obstructive pulmonary disease admitted to Zagazig university hospitals, Egypt. Egyptian Journal of Chest Diseases and Tuberculosis 2015;64:35-40. 
41 Celli BR, Cote CG, Marin JM, et al. The body-mass index, airflow obstruction, dyspnea, and exercise capacity index in chronic obstructive pulmonary disease. N Engl J Med 2004;350:1005-12.

42 Steer J, Gibson GJ, Bourke SC. Predicting outcomes following hospitalization for acute exacerbations of COPD. QJM 2010;103:817-29.

43 Shorr AF, Sun X, Johannes RS, et al. Predicting the need for mechanical ventilation in acute exacerbations of chronic obstructive pulmonary disease: comparing the CURB-65 and BAP-65 scores. $J$ Crit Care 2012;27:564-70.

44 Patil SP, Krishnan JA, Lechtzin N, et al. In-Hospital mortality following acute exacerbations of chronic obstructive pulmonary disease. Arch Intern Med 2003;163:1180-6.
45 Mach K. [Staphylococcus epidermidis infection. Results of three groups evaluated according to APACHE II--severity of disease classification system--with reference to risk, mortality and prognosis]. Wien Klin Wochenschr 1992;104:540-2.

46 Akhter S, Warraich UA, Ghazal S, et al. Assessment and comparison of APACHE II (acute physiology and chronic health evaluation), SOFA (sequential organ failure assessment) score and curb 65 (confusion; urea; respiratory rate; blood pressure), for prediction of inpatient mortality in acute exacerbation of chronic obstructive pulmonary disease. J Pak Med Assoc 2019;69:211-5.

47 Costello RW, Cushen B. A risk stratification tool for exacerbations of COPD: time to switch to DECAF. Thorax 2016;71:489-90.

48 Irwig L, Macaskill P, Glasziou P, et al. Meta-Analytic methods for diagnostic test accuracy. J Clin Epidemiol 1995;48:119-30. 